\title{
Theory of lepton universality, flavor and number violation
}

\author{
Paride Paradisi* Dipartimento di Fisica e Astronomia 'G. Galilei', Università di \\ Padova, Italy \\ Istituto Nazionale Fisica Nucleare, Sezione di Padova, I-35131 Padova, Italy \\ E-mail: paride.paradisi@pd.infn.it
}

\begin{abstract}
Lepton flavour universality (LFU) in B-decays is revisited by considering a class of semileptonic operators defined at a scale $\Lambda$ above the electroweak scale $v$. The importance of quantum effects is emphasised [1]. We construct the low-energy effective Lagrangian taking into account the running effects from $\Lambda$ down to $v$ through the one-loop renormalization group equations (RGE) in the limit of exact electroweak symmetry and QED RGEs from $v$ down to the $1 \mathrm{GeV}$ scale. The most important quantum effects turn out to be the modification of the leptonic couplings of the vector boson $Z$ and the generation of a purely leptonic effective Lagrangian. Large LFU breaking effects in $Z$ and $\tau$ decays as well as visible lepton flavour violating (LFV) effects in $\tau$ decays are induced.
\end{abstract}

9th International Workshop on the CKM Unitarity Triangle

28 November - 3 December 2016

Tata Institute for Fundamental Research (TIFR), Mumbai, India

\footnotetext{
* Speaker.
} 


\section{Introduction}

Lepton flavour universality (LFU) tests are among the most powerful probes of the Standard Model (SM) and, in turn, of New Physics (NP) effects. In recent years, experimental data in $B$ physics hinted at deviations from the SM expectations, both in charged-current as well as neutralcurrent transitions. The statistically most significant data are:

- A $3.9 \sigma$ violation from the $\tau / \ell$ universality $(\ell=\mu, e)$ in the charged-current $b \rightarrow c$ decays [2, $3,4,5]$ :

$$
\begin{aligned}
R_{D^{(*)}}^{\tau / \ell} & =\frac{\mathscr{B}\left(\bar{B} \rightarrow D^{(*)} \tau \bar{v}\right)_{\exp } / \mathscr{B}\left(\bar{B} \rightarrow D^{(*)} \tau \bar{v}\right)_{\mathrm{SM}}}{\mathscr{B}\left(\bar{B} \rightarrow D^{(*)} \ell \bar{v}\right)_{\exp } / \mathscr{B}\left(\bar{B} \rightarrow D^{(*)} \ell \bar{v}\right)_{\mathrm{SM}}}, \\
R_{D}^{\tau / \ell} & =1.37 \pm 0.17, \quad R_{D^{*}}^{\tau / \ell}=1.28 \pm 0.08 .
\end{aligned}
$$

- A $2.6 \sigma$ deviation from $\mu / e$ universality in the neutral-current $b \rightarrow s$ transition [6]:

$$
R_{K}^{\mu / e}=\frac{\mathscr{B}\left(B \rightarrow K \mu^{+} \mu^{-}\right)_{\exp }}{\mathscr{B}\left(B \rightarrow K e^{+} e^{-}\right)_{\exp }}=0.745_{-0.074}^{+0.090} \pm 0.036
$$

while $\left(R_{K}^{\mu / e}\right)_{S M}=1$ up to few $\%$ corrections [7].

As argued in [8] by means of global-fit analyses, the explanation of the $R_{K}^{\mu / e}$ anomaly favours an effective 4 -fermion operator involving left-handed currents, $\left(\bar{s}_{L} \gamma_{\mu} b_{L}\right)\left(\bar{\mu}_{L} \gamma_{\mu} \mu_{L}\right)$. This naturally suggests to account also for the charged-current anomaly through a left-handed operator $\left(\bar{c}_{L} \gamma_{\mu} b_{L}\right)\left(\bar{\tau}_{L} \gamma_{\mu} v_{L}\right)$ which is related to $\left(\bar{s}_{L} \gamma_{\mu} b_{L}\right)\left(\bar{\mu}_{L} \gamma_{\mu} \mu_{L}\right)$ by the $S U(2)_{L}$ gauge symmetry [9]. Clearly, this picture might work only provided NP couples much more strongly to the third generation than to the first two.

In ref. [1], we revisited the LFU in B-decays focusing on a class of semileptonic operators defined above the electroweak scale $v$ and invariant under the full SM gauge group, along the lines of Refs. $[9,10,11,12,13]$. The main new development of our study is the construction of the low-energy effective Lagrangian taking into account the running of the Wilson coefficients of a suitable operator basis and the matching conditions when mass thresholds are crossed. The running effects from the NP scale $\Lambda$ down to the electroweak scale are included through the one-loop renormalization group equations (RGE) in the limit of exact electroweak symmetry [14]. From the electroweak scale down to the $1 \mathrm{GeV}$ scale we use the QED RGEs. The most important quantum effects turn out to be the modification of the leptonic couplings of the vector boson $Z$ and the generation of a purely leptonic effective Lagrangian. As a result, large LFV and LFU breaking effects in $Z$ and $\tau$ decays are induced.

\section{Effective Lagrangians}

If the NP contributions originate at a scale $\Lambda \gg v$, in the energy window above $v$ and below $\Lambda$ the NP effects can be described by an effective Lagrangian $\mathscr{L}=\mathscr{L}_{\mathrm{SM}}+\mathscr{L}_{\mathrm{NP}}$ invariant under the SM gauge group. Here we assume that NP is dominated by

$$
\mathscr{L}_{\mathrm{NP}}=\frac{C_{1}}{\Lambda^{2}}\left(\bar{q}_{3 L} \gamma^{\mu} q_{3 L}\right)\left(\bar{\ell}_{3 L} \gamma_{\mu} \ell_{3 L}\right)+\frac{C_{3}}{\Lambda^{2}}\left(\bar{q}_{3 L} \gamma^{\mu} \tau^{a} q_{3 L}\right)\left(\bar{\ell}_{3 L} \gamma_{\mu} \tau^{a} \ell_{3 L}\right)
$$


We move from the interaction to the mass basis through the unitary transformations $u_{L} \rightarrow V_{u} u_{L}$, $d_{L} \rightarrow V_{d} d_{L}, V_{u}^{\dagger} V_{d}=V, v_{L} \rightarrow U_{e} v_{L}, e_{L} \rightarrow U_{e} e_{L}$ where $V$ is the CKM matrix and neutrino masses have been neglected. For future convenience we define

$$
\lambda_{i j}^{q}=V_{q 3 i}^{*} V_{q 3 j} \quad \lambda_{i j}^{e}=U_{e 3 i}^{*} U_{e 3 j} \quad \lambda_{i j}^{u d}=V_{u 3 i}^{*} V_{d 3 j},
$$

with $q=u, d$. Starting from the effective Lagrangian $\mathscr{L}_{\mathrm{NP}}$ at the scale $\Lambda$, at lower energies an effective Lagrangian is induced by RGE and by integrating out the heavy degrees of freedom.

The effective Lagrangian describing the semileptonic processes $b \rightarrow s \ell \ell$ and $b \rightarrow s v v$ is

$$
\mathscr{L}_{\text {eff }}^{\mathrm{NC}}=\frac{4 G_{F}}{\sqrt{2}} \lambda_{b s}\left(C_{v}^{i j} \mathscr{O}_{v}^{i j}+C_{9}^{i j} \mathscr{O}_{9}^{i j}+C_{10}^{i j} \mathscr{O}_{10}^{i j}\right)+\text { h.c. },
$$

where $\lambda_{b s}=V_{t b} V_{t s}^{*}$ and the operators $\mathscr{O}_{v}$ and $\mathscr{O}_{9,10}$ read

$$
\begin{aligned}
& \mathscr{O}_{v}^{i j}=\frac{e^{2}}{(4 \pi)^{2}}\left(\bar{s}_{L} \gamma_{\mu} b_{L}\right)\left(\bar{v}_{i} \gamma^{\mu}\left(1-\gamma_{5}\right) v_{j}\right), \\
& \mathscr{O}_{9}^{i j}=\frac{e^{2}}{(4 \pi)^{2}}\left(\bar{s}_{L} \gamma_{\mu} b_{L}\right)\left(\bar{e}_{i} \gamma^{\mu} e_{j}\right), \quad \mathscr{O}_{10}^{i j}=\frac{e^{2}}{(4 \pi)^{2}}\left(\bar{s}_{L} \gamma_{\mu} b_{L}\right)\left(\bar{e}_{i} \gamma^{\mu} \gamma_{5} e_{j}\right) .
\end{aligned}
$$

By matching $\mathscr{L}_{\text {eff }}^{\mathrm{NC}}$ with $\mathscr{L}_{\mathrm{NP}}$, we obtain:

$$
C_{9}^{i j} \simeq-C_{10}^{i j} \simeq \frac{4 \pi^{2}}{e^{2} \lambda_{b s}} \frac{v^{2}}{\Lambda^{2}}\left(C_{1}+C_{3}\right) \lambda_{23}^{d} \lambda_{i j}^{e}, \quad C_{v}^{i j} \simeq \frac{4 \pi^{2}}{e^{2} \lambda_{b s}} \frac{v^{2}}{\Lambda^{2}}\left(C_{1}-C_{3}\right) \lambda_{23}^{d} \lambda_{i j}^{e},
$$

The effective Lagrangian relevant for charged-current processes like $b \rightarrow c \ell v$ is given by

$$
\mathscr{L}_{\text {eff }}^{\mathrm{cc}}=-\frac{4 G_{F}}{\sqrt{2}} V_{c b}\left(C_{L}^{c b}\right)_{i j}\left(\bar{c}_{L} \gamma_{\mu} b_{L}\right)\left(\bar{e}_{L i} \gamma^{\mu} v_{L j}\right)+\text { h.c. }
$$

where the coefficient $\left(C_{L}^{c b}\right)_{i j}$ reads

$$
\left(C_{L}^{c b}\right)_{i j}=\delta_{i j}-\frac{v^{2}}{\Lambda^{2}} \frac{\lambda_{23}^{u d}}{V_{c b}} C_{3} \lambda_{i j}^{e}
$$

One of the effects due to $\mathscr{L}_{\mathrm{NP}}$ is the modification of the leptonic couplings of the vector bosons $W$ and $Z$. Focusing on the $Z$ couplings, we find that

$$
\mathscr{L}_{Z}=\frac{g_{2}}{c_{W}} \bar{e}_{i}\left(\not Z g_{\ell L}^{i j} P_{L}+\not g_{\ell R}^{i j} P_{R}\right) e_{j}+\frac{g_{2}}{c_{W}} \bar{v}_{L i} \not Z g_{v L}^{i j} v_{L j},
$$

where $g_{f L, R}=g_{f L, R}^{\mathrm{SM}}+\Delta g_{f L, R}, c_{W}=\cos \theta_{W}$ and

$$
\begin{aligned}
\Delta g_{\ell L}^{i j} & \simeq \frac{v^{2}}{\Lambda^{2}}\left(3 y_{t}^{2} c_{-} \lambda_{33}^{u} L_{t}+g_{2}^{2} C_{3} L_{z}+\frac{g_{1}^{2}}{3} C_{1} L_{z}\right) \frac{\lambda_{i j}^{e}}{16 \pi^{2}}, \\
\Delta g_{v L}^{i j} & \simeq \frac{v^{2}}{\Lambda^{2}}\left(3 y_{t}^{2} c_{+} \lambda_{33}^{u} L_{t}-g_{2}^{2} C_{3} L_{z}+\frac{g_{1}^{2}}{3} C_{1} L_{z}\right) \frac{\lambda_{i j}^{e}}{16 \pi^{2}},
\end{aligned}
$$

with $L_{t}=\log \left(\Lambda / m_{t}\right), L_{z}=\log \left(\Lambda / m_{Z}\right)$ and $\Delta g_{\ell R}=0$. Quantum effects generate also a purely leptonic effective Lagrangian, as well as corrections to the semileptonic interactions:

$$
\begin{aligned}
\mathscr{L}_{\mathrm{eff}}^{\ell}= & -\frac{4 G_{F}}{\sqrt{2}} \lambda_{i j}^{e}\left[\left(\bar{e}_{L i} \gamma_{\mu} e_{L j}\right) \sum_{\psi} \bar{\psi} \gamma^{\mu} \psi\left(2 g_{\psi}^{z} c_{t}^{e}-Q_{\psi} c_{\gamma}^{e}\right)\right. \\
& \left.++c_{t}^{c c}\left(\bar{e}_{L i} \gamma_{\mu} v_{L j}\right)\left(\bar{v}_{L k} \gamma^{\mu} e_{L k}+\bar{u}_{L k} \gamma^{\mu} V_{k l} d_{L l}\right)+\text { h.c. }\right],
\end{aligned}
$$


where $\psi=\left\{v_{L k}, e_{L k, R k}, u_{L, R}, d_{L, R}, s_{L, R}\right\}$ and $g_{\psi}^{z}=T_{3}(\psi)-Q_{\psi} \sin ^{2} \theta_{W}$. Finally, the coefficients $c_{t}^{e, c c}$ and $c_{\gamma}^{e}$ are given by

$$
\begin{aligned}
& c_{\gamma}^{e}=\frac{e^{2}}{48 \pi^{2}} \frac{v^{2}}{\Lambda^{2}}\left[\left(3 C_{3}-C_{1}\right) \log \frac{\Lambda^{2}}{\mu^{2}}-\left(C_{1}+C_{3}\right) \lambda_{33}^{d} \log \frac{m_{b}^{2}}{\mu^{2}}+2\left(C_{1}-C_{3}\right)\left(\lambda_{33}^{u} \log \frac{m_{t}^{2}}{\mu^{2}}+\lambda_{22}^{u} \log \frac{m_{c}^{2}}{\mu^{2}}\right)\right], \\
& c_{t}^{e}=\frac{3 y_{t}^{2}}{32 \pi^{2}} \frac{v^{2}}{\Lambda^{2}}\left(C_{1}-C_{3}\right) \lambda_{33}^{u} \log \frac{\Lambda^{2}}{m_{t}^{2}}, \quad c_{t}^{c c}=\frac{3 y_{t}^{2}}{16 \pi^{2}} \frac{v^{2}}{\Lambda^{2}} C_{3} \lambda_{33}^{u}\left[\log \frac{\Lambda^{2}}{m_{t}^{2}}+\frac{1}{2}\right] .
\end{aligned}
$$

The residual scale dependence is removed by evaluating the matrix elements in the low energy theory. For simplicity, we have done this within the quark model, by assuming for $u, d$ and $s$ a common constituent mass $\mu \approx 1 \mathrm{GeV}$.

\section{Observables}

In our model, $R_{K}^{\mu / e}$ is approximated by the expression

$$
R_{K}^{\mu / e} \approx \frac{\left|C_{9}^{\mu \mu}+C_{9}^{\mathrm{SM}}\right|^{2}}{\left|C_{9}^{e e}+C_{9}^{\mathrm{SM}}\right|^{2}} \approx 1-0.28 \frac{\left(C_{1}+C_{3}\right)}{\Lambda^{2}(\mathrm{TeV})} \frac{\lambda_{23}^{d} \lambda_{22}^{e}}{10^{-3}}
$$

while the expression for $R_{D^{(*)}}^{\tau / \ell}$ reads

$$
R_{D^{(*)}}^{\tau / \ell}=\frac{\sum_{j}\left|\left(C_{L}^{c b}\right)_{3 j}\right|^{2}}{\sum_{j}\left|\left(C_{L}^{c b}\right)_{\ell j}\right|^{2}} \approx 1-\frac{0.12 C_{3}}{\Lambda^{2}(\mathrm{TeV})}\left(\lambda_{33}^{d}+\frac{V_{c s}}{V_{c b}} \lambda_{23}^{d}\right) .
$$

Non trivial constraints arise from the observable $R_{K}^{v v}=\mathscr{B}(B \rightarrow K v \bar{v}) / \mathscr{B}(B \rightarrow K v \bar{v})_{\mathrm{SM}}$ [13],

$$
R_{K}^{v v}=\frac{\sum_{i j}\left|C_{V}^{\mathrm{SM}} \delta^{i j}+C_{v}^{i j}\right|^{2}}{3\left|C_{V}^{\mathrm{SM}}\right|^{2}} \approx 1+\frac{0.6 c_{-}}{\Lambda^{2}(\mathrm{TeV})}\left(\frac{\lambda_{23}^{d}}{0.01}\right)+\frac{0.3 c_{-}^{2}}{\Lambda^{4}(\mathrm{TeV})}\left(\frac{\lambda_{23}^{d}}{0.01}\right)^{2},
$$

while the experimental bound reads $R_{K}^{v v}<4.3$ [15]. If LFU effects arise from LFV sources, LFV phenomena are unavoidable [11]. In our setting, it turns out that

$$
\mathscr{B}(B \rightarrow K \tau \mu) \approx 4 \times 10^{-8}\left|C_{9}^{\mu \tau}\right|^{2} \approx 10^{-7}\left|\frac{C_{9}^{\mu \mu}}{0.5} \frac{0.3}{\lambda_{23}^{e}}\right|^{2}
$$

which is orders of magnitude below the current bound $\mathscr{B}(B \rightarrow K \tau \mu) \leq 4.8 \times 10^{-5}$ [16].

Modifications of the leptonic $Z$ couplings are constrained by the LEP measurements [17]

$$
\frac{v_{\tau}}{v_{e}}=0.959(29), \quad \frac{a_{\tau}}{a_{e}}=1.0019(15)
$$

where $v_{\ell}=g_{\ell L}^{\ell \ell}+g_{\ell R}^{\ell \ell}$ and $a_{\ell}=g_{\ell L}^{\ell \ell}-g_{\ell R}^{\ell \ell}$ are the vector and axial-vector couplings, respectively, which in our model read

$$
\begin{aligned}
& \frac{v_{\tau}}{v_{e}} \simeq 1-\frac{2 \Delta g_{\ell L}^{33}}{\left(1-4 s_{W}^{2}\right)} \approx 1-0.05 \frac{\left(c_{-}+0.2 C_{3}\right)}{\Lambda^{2}(\mathrm{TeV})} \\
& \frac{a_{\tau}}{a_{e}} \simeq 1-2 \Delta g_{\ell L}^{33} \approx 1-0.004 \frac{\left(c_{-}+0.2 C_{3}\right)}{\Lambda^{2}(\mathrm{TeV})}
\end{aligned}
$$


Moreover, modifications of the $Z$ couplings to neutrinos affect the extraction of the number of neutrinos $N_{v}$ from the invisible $\mathrm{Z}$ decay width. We find that

$$
N_{v}=2+\left(\frac{g_{v L}^{33}}{g_{v L}^{S M}}\right)^{2} \simeq 3+4 \Delta g_{v L}^{33} \approx 3+0.008 \frac{\left(c_{+}-0.2 C_{3}\right)}{\Lambda^{2}(\mathrm{TeV})}
$$

to be compared with the experimental result $N_{v}=2.9840 \pm 0.0082$ [17].

LFU breaking effects in $\tau \rightarrow \ell \bar{v} v$ (with $\ell_{1,2}=e, \mu$ ) are described by the observables

$$
R_{\tau}^{\tau / \ell_{1,2}}=\frac{\mathscr{B}\left(\tau \rightarrow \ell_{2,1} v \bar{v}\right)_{\exp } / \mathscr{B}\left(\tau \rightarrow \ell_{2,1} v \bar{v}\right)_{\mathrm{SM}}}{\mathscr{B}(\mu \rightarrow e v \bar{v})_{\exp } / \mathscr{B}(\mu \rightarrow e v \bar{v})_{\mathrm{SM}}} \approx 1+2 c_{t}^{c c} \lambda_{33}^{e} \approx 1+\frac{0.008 C_{3}}{\Lambda^{2}(\mathrm{TeV})}
$$

and are experimentally tested at the few per-mill level [18]

$$
R_{\tau}^{\tau / \mu}=1.0022 \pm 0.0030, R_{\tau}^{\tau / e}=1.0060 \pm 0.0030
$$

The effective Lagrangian of eq. (2.12) generates LFV processes such as $\tau \rightarrow \mu \ell \ell$ and $\tau \rightarrow \mu P$ with
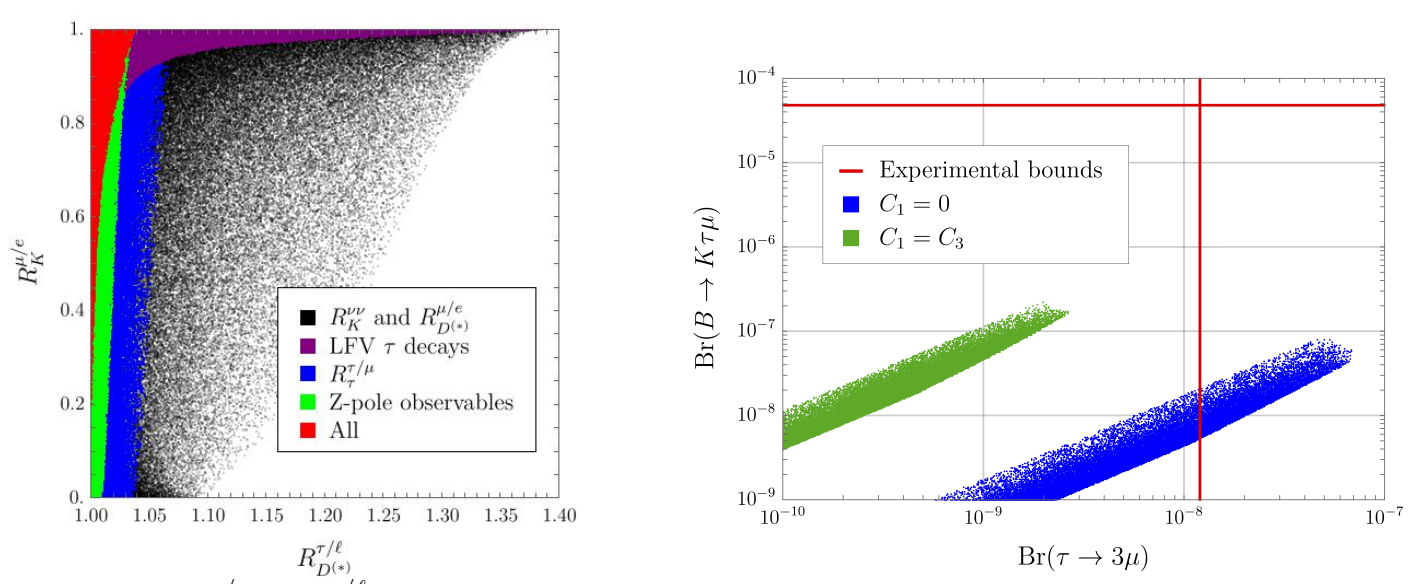

Figure 1: Left: $R_{K}^{\mu / e}$ vs. $R_{D^{(*)}}^{\tau / \ell}$ for $C_{1}=0,\left|C_{3}\right| \leq 3,\left|\lambda_{23}^{d}\right| \leq 0.04$ and $\left|\lambda_{23}^{e}\right| \leq 1 / 2$. The allowed regions are coloured according to the legend. Right: $\mathscr{B}(B \rightarrow K \tau \mu)$ vs. $\mathscr{B}(\tau \rightarrow 3 \mu)$ for $\left|\lambda_{23}^{d}\right|=0.01, C_{1}=C_{3}$ (green points) or $C_{1}=0$ (blue points) imposing all the experimental bounds except $R_{D^{(*)}}^{\tau / \ell}$.

$P=\pi, \eta, \eta^{\prime}, \rho$, etc. The most sensitive channels, taking into account their NP sensitivities and experimental resolutions, are $\tau \rightarrow \mu \ell \ell, \tau \rightarrow \mu \rho$ and $\tau \rightarrow \mu \pi$. For instance, we find

$$
\mathscr{B}(\tau \rightarrow 3 \mu) \approx 5 \times 10^{-8} \frac{c_{-}^{2}}{\Lambda^{4}(\mathrm{TeV})}\left(\frac{\lambda_{23}^{e}}{0.3}\right)^{2}
$$

where the current bounds are $\mathscr{B}(\tau \rightarrow 3 \mu) \leq 1.2 \times 10^{-8}[16]$.

Most importantly, we find that $R_{\tau}^{\tau / \ell}$ strongly disfavours an explanation of the $R_{D^{(*)}}^{\tau / \ell}$ anomaly, see the left plot of fig. 1 . In the right plot of fig. 1 , we show $\mathscr{B}(B \rightarrow K \tau \mu)$ vs. $\mathscr{B}(\tau \rightarrow 3 \mu)$. Considering the current and expected future experimental sensitivities, we conclude that $\tau \rightarrow 3 \mu$ is a more powerful probe than $B \rightarrow K \tau \mu$. 


\section{Conclusions}

Recent experimental data hinting at non-standard LFU breaking effects in semileptonic $B$ decays stimulated many theoretical investigations of NP scenarios. In ref. [1], we revisited LFU in B-decays assuming a class of gauge invariant semileptonic operators at the NP scale $\Lambda \gg v$, as in Refs. $[9,10,11,12,13]$. We constructed the low-energy effective Lagrangian taking into account the running effects from $\Lambda$ down to $v$ through the one-loop RGEs in the limit of exact electroweak symmetry and QED RGEs from $v$ down to the $1 \mathrm{GeV}$ scale. At the quantum level, we find that the leptonic couplings of the $W$ and $Z$ vector bosons are modified. Moreover, quantum effects generate also a purely leptonic effective Lagrangian, as well as corrections to the semileptonic interactions. The main phenomenological implications of these findings are the generation of large LFU breaking effects in $Z$ and $\tau$ decays, which are correlated with the B-anomalies, and $\tau$ LFV processes. Overall, the experimental bounds on $Z$ and $\tau$ decays significantly constrain LFU breaking effects in B-decays, challenging an explanation of the current non-standard data. Interestingly, if LFU breaking effects arise from LFV sources, the most sensitive LFV channels are not $B$-decays, as commonly claimed in the literature but, instead, $\tau$ decays such as $\tau \rightarrow \mu \ell \ell$ and $\tau \rightarrow \mu \rho$. Although our results have been obtained in the context of an effective Lagrangian dominated by left-handed operators, the present work shows that electroweak radiative effects should be carefully analysed in any framework addressing the explanation of B-anomalies.

\section{References}

[1] F. Feruglio, P. Paradisi and A. Pattori, Phys. Rev. Lett. 118 (2017) no.1, 011801.

[2] J. P. Lees et al. [BaBar Collaboration], Phys. Rev. D 88 (2013) 7, 072012

[3] M. Huschle et al. [Belle Collaboration], Phys. Rev. D 92 (2015) 7, 072014.

[4] R. Aaij et al. [LHCb Collaboration], Phys. Rev. Lett. 115 (2015) 15, 159901.

[5] S. Fajfer, J. F. Kamenik and I. Nisandzic, Phys. Rev. D 85 (2012) 094025.

[6] R. Aaij et al. [LHCb Collaboration], Phys. Rev. Lett. 113 (2014) 151601.

[7] M. Bordone, G. Isidori and A. Pattori, Eur. Phys. J. C 76 (2016) no.8, 440.

[8] W. Altmannshofer and D. M. Straub, Eur. Phys. J. C 75 (2015) no.8, 382.

[9] B. Bhattacharya et al., Phys. Lett. B 742 (2015) 370.

[10] S. Fajfer, J. F. Kamenik, I. Nisandzic and J. Zupan, Phys. Rev. Lett. 109 (2012) 161801.

[11] S. L. Glashow, D. Guadagnoli and K. Lane, Phys. Rev. Lett. 114 (2015) 091801.

[12] R. Alonso, B. Grinstein and J. Martin Camalich, JHEP 1510 (2015) 184.

[13] L. Calibbi, A. Crivellin and T. Ota, Phys. Rev. Lett. 115 (2015) 181801.

[14] E. E. Jenkins, A. V. Manohar and M. Trott, JHEP 1401 (2014) 035. R. Alonso, E. E. Jenkins, A. V. Manohar and M. Trott, JHEP 1404 (2014) 159.

[15] J. P. Lees et al. [BaBar Collaboration], Phys. Rev. D 87 (2013) no.11, 112005.

[16] Y. Amhis et al. [Heavy Flavor Averaging Group (HFAG) Collaboration], arXiv:1412.7515 [hep-ex].

[17] K. A. Olive et al. [Particle Data Group Collaboration], Chin. Phys. C 38 (2014) 090001.

[18] A. Pich, Prog. Part. Nucl. Phys. 75 (2014) 41. 